\title{
Impacto y consecuencias sociales contemporáneas de las políticas de tutela estatal en las reservas indígenas canadienses ${ }^{*}$
}

\author{
Alfonso Marquina Márquez \\ Universidad de Granada \\ ammarq@ugr.es \\ Raúl Ruiz Callado \\ Universidad de Alicante \\ raulruiz@ua.es \\ Jorge Virchez \\ University of Laurentian \\ jvirchez@laurentian.ca
}

Resumen: Este artículo tiene como objetivo examinar el impacto y las consecuencias sociales contemporáneas del mantenimiento del sistema de organización social denominado «reserva indígena» en Canadá. Mediante un estudio de caso, se explora cómo la politica organizacional y la estructura institucional contemporánea en las reservas indígenas canadienses están alimentando problemas psicosociales y de salud mental como, por ejemplo, abuso de sustancias psicoactivas, depresión, negligencia parental o violencia doméstica. Tres características estructurales se identifican como problemas inherentes en la estructura organizacional de las reservas contemporáneas: el desempleo estructural, la dependencia de programas de asistencia social y el faccionalismo comunitario.

\footnotetext{
*Este trabajo forma parte de dos proyectos de investigación: I+D+i SEJ2005-09344/SOCI, financiado por el Ministerio de Educación y Ciencia (España), e I+D+i CURA/NORTHERN, financiado por el Social Sciences and Humanities Research Council (Canadá). Las ideas y materiales aquí expuestos han sido debatidos en un seminario de trabajo en la Universidad de Alicante. Los autores agradecen el apoyo institucional del grupo de investigación de Estudios Socioculturales Aplicados de la Universidad de Granada, el Centre for Research in Social Justice and Policy en la Laurentian University y al Centre for Research on Immigration, Ethnicity and Citizenship (CRIEC) de la Université du Québec à Montréal (UQAM). Finalmente, los autores también desean agradecer al evaluador externo de RIO sus ideas y comentarios constructivos.
} 
Palabras clave: politica organizacional, faccionalismo, salud mental, sistema de reserva indigena, Canadá.

\begin{abstract}
This paper aims to examine the impact and contemporary social consequences of the maintenance of the organizational system known as Indian reserve in Canada. By means of a case study this paper explores how contemporary organizational politics and the institutional structure of the Canadian Indian reserves are giving rise to psychosocial and mental health problems such as drug abuse, depression, parental negligence, domestic violence, etc. Three structural characteristics are identified as inberent problems to the organizational structure within contemporary Canadian native reserves: structural unemployment, dependence on social assistance programs, and community factionalism.
\end{abstract}

Keywords: organizational politics, factionalism, health mental, indigenous reserve system, Canada. 


\section{Introducción}

En Canadá, el denominado «sistema de reserva indígena» ha sido un artefacto legal cuyo propósito fundamental fue establecer la asimilación de las poblaciones indígenas dentro de la vida económica, industrial y social canadiense, transformando las agrupaciones familiares y subtribales flexibles de caza del periodo poscontacto en comunidades sedentarias rígidamente definidas (Flanagan, 2008; Tanner, 2008; Kirmayer y Valaskakis, 2008). La sedentarización de las poblaciones indígenas ocurrió mediante la aplicación del sistema de reserva, como un nuevo modelo de organización social establecido por el gobierno federal bajo la aprobación del Acta India en 1876. Esta ley consistía en un conjunto de medidas administrativas para regular jurídicamente la vida social, económica y política de las poblaciones indígenas canadienses. Esta nueva legislación jurídica otorgaba control institucional para gobernar sobre casi todos los aspectos de la vida indígena: la naturaleza del gobierno en las reservas, los sistemas de tenencia de la tierra y el establecimiento de restricciones sobre prácticas culturales ancestrales (Coates, 2008; Wadden, 2008; Flanagan, 2008). Desde la aprobación de la ley indígena, indian reserve llegó a ser el término legal para cualquier agrupación indígena, adquiriendo de esta forma una existencia legal. Cada reserva indígena debía contar con una lista de miembros afiliados a la reserva, un territorio asignado y una organización política basada en un jefe y un consejo de banda que dependían del agente indígena como brazo administrativo del gobierno canadiense.

Esta política pública de asentamientos permanentes para las poblaciones indígenas puede ser contemplada como un ejemplo ilustrativo de ingeniería social contemporánea, es decir, como un mecanismo administrativo y de organización social con propósitos asimilacionistas (Scott, 1998; Tanner, 2008). Irónicamente, la política pública de extender la «modernidad» a poblaciones indígenas, mediante el establecimiento de asentamientos permanentes, participación en la economía industrial, empleo asalariado y acceso a servicios sociales, ha tenido consecuencias no intencionadas. Hoy día, las poblaciones indígenas canadienses ${ }^{1}$ que residen en un contexto de reserva continúan teniendo los peores resultados de salud, comparadas con el resto de grupos étnicos que constituyen el estado multicultural canadiense (Chiefs of Ontario, 2010; King et alii, 2009; Kirmayer y Valaskakis, 2008; Health Canada, 2008; Waldram et alii, 2006; Jones, 2004). Aunque ha habido una mejora sustancial en los últimos años, sigue existiendo

1 Aboriginal people es el término legal empleado desde 1982 en la Constitución canadiense para referirse a la población de ascendencia indígena. En la actualidad aboriginal people representa tres subcategorías culturales: first nations (indios), inuits (esquimales) y metis (descendientes de indios y comerciantes de pieles). La población indígena canadiense representa el 3,8\% (1.200.000 individuos) de la población total canadiense. En la actualidad hay cerca de 700 reservas indígenas (INAC, 2010). 
una brecha en la esperanza de vida entre la población de ascendencia indígena y el resto de la población canadiense. ${ }^{2}$ Estas poblaciones de ascendencia indígena tienen mayor incidencia de tuberculosis, son más propensas a padecer diabetes, sufren más enfermedades del corazón e hipertensión y registran índices más altos de discapacidad (Kirmayer y Valaskakis, 2008; Waldram et alii, 2006).

Actualmente, existe un debate público y académico acerca del impacto del sistema de reserva indígena, como organización social y administrativa, sobre las patologías sociales modernas que sufren gran parte de las comunidades indígenas contemporáneas: abuso de sustancias psicoactivas, adicciones, suicidios, violencia doméstica, negligencia parental, abuso sexual... (Vírchez y Faucheux, 2012; Yahn, 2009; Wadden, 2008; Barsh, 2008; Flanagan, 2008; Kirmayer y Valaskakis, 2008; Tanner, 2008; Adelson, 2004; Jones, 2004; Quintero, 2002; Berman, 2003; Fast, 2002; Carstens, 2000; Warry, 1998; Brady, 1995; Moscovitch y Webster, 1995; Dixon y Scheurell, 1995; Dyck y Waldram, 1993). Se han expuesto algunas explicaciones sobre este fenómeno generalizado en las reservas indígenas, pero el debate continúa abierto y es bastante controvertido (Tanner, 2008). Entre los factores explicativos expuestos en la literatura académica canadiense sobre el aumento de patologías sociales se encuentran: el rápido cambio social, la asimilación forzosa, el impacto social de la pobreza e industrialización, la desposesión de la tierra, el asentamiento dirigido, la pérdida de continuidad cultural, la carencia de trabajo significativo y el impacto del sistema escolar asimilacionista conocido como residential school (Vírchez y Faucheux, 2012; Tanner, 2008).

Por otra parte, las políticas poscoloniales de reconciliación, la conquista de mayor autogobierno indígena y el despegue de un desarrollo institucional experimentado en las reservas indígenas desde 1980 están produciendo como resultado otro conjunto de contradicciones y consecuencias no intencionadas. Por un lado, las políticas de autogobierno han generado una clase social indígena emergente profesional, bien remunerada y con aspiraciones sociopolíticas. Por otro lado, el mantenimiento de políticas de discriminación positiva dentro de las reservas ha generado una infraclase socioeconómica, sin estudios, con desempleo estructural y fuertemente dependiente de programas de asistencia social. Politólogos como Flanagan (2008), sociólogos como Thatcher (2004) o antropólogos como Carstens (2000) han señalado que, en realidad, el autogobierno indígena es una forma encubierta de asistencia social. El autogobierno como estructura organizativa tiene problemas inherentes cuyos efectos están llegando a ser más visibles cuanto más dinero y poder es concedido al consejo de banda que gobierna las reservas

2 Según Kirmayer et alii (2008), utilizando datos oficiales publicados por Health Canada, la esperanza de vida para varones de grupos culturales first nations sería de 70,4 años, frente a 77,1 años para el resto de la población. Las cifras correspondientes para las mujeres se situaría en 75,5 frente a 82,2. 
indígenas. El sistema de reserva indígena está asolado con características estructurales que animan más que constriñen la desigualdad dentro de este modelo de organización social.

Este trabajo tiene como objetivo examinar el impacto, las contradicciones $y$ las consecuencias sociales contemporáneas del mantenimiento de esta forma organizacional, social y administrativa denominada reserva indígena en Canadá. ¿Qué características tienen en común las comunidades organizadas bajo el sistema de reserva para que durante décadas mantengan mayores índices de patologías sociales que el resto de comunidades rurales con estructuras económicas y demográficas similares a las reservas? El artículo utiliza como marco teórico la economía política de la planificación social, desarrollada por el científico social James C. Scott en sus ensayos académicos Seeing Like a State: How Certain Schemes to Improve the Human Condition Have Failed (1998) y The Art of Not Being Governed (2009). El marco teórico de Scott tiene una enorme relevancia para comprender, desde un punto de vista sociológico, las condiciones ideológicas de los fracasos de planificación estatal desarrollados a lo largo de los siglos XIX y xx. Scott sitúa el desarrollo de la ingeniería social de la planificación estatal en la ideología del «alto modernismo»: «las creencias en el progreso técnico y científico que fue asociado con la industrialización en Europa Occidental y en Norteamérica desde aproximadamente 1830 hasta la Primera Guerra Mundial [...] y la suprema autoconfianza sobre el progreso lineal continuado, el desarrollo de conocimiento científico y técnico, la expansión de la producción, el diseño racional de orden social, la satisfacción creciente de necesidades humanas y un control creciente sobre la naturaleza» (Scott, :89).

Mediante un estudio de caso, se examinan las consecuencias contemporáneas de la política gubernamental de sedentarización y organización del trabajo industrial en las reservas indígenas del noreste de Ontario, Canadá. Actualmente, el impacto de estos dos procesos macroeconómicos «dirigidos» (directed settlement) sobre individuos de ascendencia indígena algonquina (ojibwe y cree) ha generado una serie de epidemias sociales crónicas como el abuso de sustancias psicoactivas, depresión o desempleo estructural. En este sentido, políticas organizacionales como el sistema de reserva indígena canadiense deben contemplarse como un caso de planificación centralizada del trabajo industrial con grupos indígenas, que refleja los ideales sociales y la retórica del modernismo liberal desde finales del siglo xix. La organización social y la estructura institucional contemporánea en las reservas indígenas canadienses, primero diseñadas por planificadores gubernamentales coloniales y en la actualidad mantenidas por individuos de ascendencia indígena, están alimentando el desempleo estructural, el aumento de 
adicciones, la dependencia de programas de asistencia social y el faccionalismo comunitario, lo que tiene como resultado mayor desigualdad social en el interior de las reservas.

\section{Metodología}

El análisis descriptivo de los datos presentados en este trabajo pertenece a un proyecto de investigación etnohistórico y etnográfico más amplio sobre problemas psicosociales llevado a cabo desde 2008 hasta 2012 por un equipo de investigación interdisciplinar en distintas reservas indígenas en el norte de Ontario, Canadá (Marquina, Ruiz-Callado y Vírchez, 2012; Vírchez y Faucheux, 2012; Vírchez y Brisbois, 2007). Desde el año 2005, el consumo abusivo de analgésicos opioides ha llegado a ser una auténtica epidemia social que se ha extendido a todas las reservas indígenas del norte de Ontario (Kelly et alii, 2011; Chiefs of Ontario, 2010; Sioux Lookout, 2009). Por ejemplo, en el área regional de Sioux Lookout, con una población de ascendencia indígena de 25.000 personas, al menos 9.000 personas son adictas a analgésicos opioides. En reservas indígenas como Fort Hope First Nation o Cat Lake First Nation, cerca del 80\% de la población en edad de trabajar tenía problemas con analgésicos opioides (NAHO, 2012).

Para este artículo se han seleccionado datos recopilados de una comunidad oji-cree llamada Omushkego First Nation como estudio de caso representativo para el análisis descriptivo de las consecuencias contemporáneas del sistema de reservas indígenas en el norte de Ontario. En esta reserva, el abuso de analgésicos opioides para fines recreativos ha llegado a ser una verdadera epidemia social, con cerca de 200 miembros asistiendo a un centro de metadona instalado en la

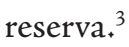

Para la recolección del material empírico se utilizó una combinación de estrategias cualitativas que son consideradas estándar en ciencias sociales: examen de fuentes documentales y archivos, observación participante, entrevistas informales, entrevistas semiestructuradas y narrativas conversacionales. ${ }^{4}$ Los datos que aparecen en la sección de resultados han sido extraídos exclusivamente del análisis de material etnohistórico y observacional. En este sentido, para el análisis descriptivo de este artículo solo se utilizan datos procedentes de una investigación de archivo y bibliográfica durante el curso académico 2010-2011 en las siguientes

\footnotetext{
3 Según estadísticas comunitarias, la reserva cuenta con 1.477 miembros registrados con indian status, pero solo residen 820 personas.

4 Para una descripción más detallada de la metodología del proyecto de investigación sobre problemas psicosociales en reservas indígenas véase Marquina, Ruiz-Callado y Vírchez (2012) y Marquina (2012).
} 
instituciones con sede en la capital de Canadá, Ottawa: Library and Archives Canada, Carleton University, Ottawa University y Aboriginal Healing Foundation. Para la descripción analítica de la segunda parte de la sección de resultados también se utilizan datos procedentes del trabajo de campo etnográfico de 11 meses realizado durante dos estancias de campo entre 2008 y 2009.

Esta investigación ha seguido las recomendaciones éticas elaboradas en 2007 por Canadian Institutes of Health Research (CIHR) a la hora de realizar trabajo de campo dentro de reservas indígenas canadienses. Este artículo sigue los principios de anonimato, privacidad y confidencialidad (artículo 5) al mantener el nombre de la reserva bajo un pseudónimo, según el acuerdo de investigación establecido con la comunidad indígena.

El contexto geográfico en el que se encuentra esta investigación es el norte de Ontario, el cual ocupa el 89\% $\left(802.378,67 \mathrm{~km}^{2}\right)$ del área total de la provincia $\left(1.076 .395 \mathrm{~km}^{2}\right)$, representando solo el 6,5\% (745.372) de la población total (13.210.667) (Dow, 2008). La parte septentrional de la provincia es única debido a su gran número de comunidades indígenas de ascendencia cree y ojibwe (Parfitt, 2006; Bones, 2003; Mawhiney y Pitblado, 1999; Coates y Morrison, 1992). Hoy en día, el norte de la provincia cuenta con 121 reservas indígenas, de las 145 reservas con las que cuenta Ontario, ${ }^{5}$ representando el 11,5\% de la población total (Yahn, 2009). Como con cualquier grupo indígena que reside en el norte de Ontario, la totalidad de los miembros contemporáneos de las reservas indígenas en esta región septentrional pertenecen a los dos grupos lingüísticos mayoritarios de habla algonquina llamados cree y ojibwe. Debido a sus vínculos lingüísticos, culturales y comerciales, ambas poblaciones, fruto del contacto continuado, conformaron un nuevo dialecto y formación étnica denominada oji-cree.

\section{Resultados}

\subsection{Transformaciones socio-históricas en la organización social indígena}

En el norte de Ontario, como en el resto de Canadá, la búsqueda y extracción de recursos naturales ha dictado históricamente los patrones de asentamiento poscontacto desde el siglo XVI entre poblaciones indígenas y colonos europeos. La demanda de recursos naturales (pieles, madera, minerales, etc.), la creación de infraestructuras de comunicación (canales, vías ferroviarias, carreteras, etc.) y el

5 Ontario es la provincia con mayor población de ascendencia indígena con un 22,7 \% del total de la población indígena canadiense (INAC, 2010). 
desarrollo de medios modernos de transporte (barcos de vapor, trenes, camiones, etc.) transformó la morfología de las regiones septentrionales de Canadá (Bones, 2003; Sherrill, 2002). Esta evolución morfológica es crítica para comprender el desarrollo de Omushkego Lake First Nation y las denominadas resource towns que emergieron en la región boreal subártica del norte de Ontario (Woodman, 2010; Dow, 2008; Mawhiney y Pitblado, 1999; Lapierre, 1992; Coates y Morrison, 1992).

Para comprender las condiciones de vida contemporáneas en reservas como Omushkego Lake First Nation y los problemas sociales que estas afrontan, necesitamos conocer algunos factores sociohistóricos determinantes que provocaron transformaciones irreversibles en el patrón de asentamiento y en la estructura organizacional de la economía política de los ancestros recientes de esta comunidad. Los impactos múltiples que los colonos europeos generaron a los grupos indígenas que residían en el norte de Ontario han significado la constante relocalización de las agrupaciones familiares indígenas durante más de trescientos años. Algunos desplazamientos fueron voluntarios como, por ejemplo, el que fue fruto de la decisión de establecerse cerca de los puestos de intercambio europeos en el siglo XVII y en las líneas comerciales ferroviarias en los siglos XIX y XX. Otros fueron involuntarios, causados por las incursiones territoriales de grupos iroqueses en el siglo xvi o del Departamento de Asuntos Indígenas del gobierno canadiense en el siglo XIX (Flanagan, 2008). El cuadro 1 resume en forma de esquema las transformaciones sociales que desde un punto de vista organizacional han experimentado las poblaciones indígenas algonquinas del norte de Ontario. A continuación, las siguientes tres secciones describen sintéticamente las transformaciones sociohistóricas de la estructura organizacional indígena.

\section{Cuadro 1. Transición socioorganizacional de las poblaciones indígenas en el norte de Ontario (Canadá).}

\begin{tabular}{|c|c|c|}
\hline Precontacto (S. XV-XVI) & Poscontacto (S. VII-XIX) & Tutela administrativa (S. $x x)$ \\
\hline $\begin{array}{l}\text { + Agrupación familiar } \\
\text { flexible de caza } \\
\text { Economía de } \\
\text { subsistencia: caza- } \\
\text { recolección } \\
\text { + Sistema de liderazgo } \\
\text { informal: Elder }\end{array}$ & $\begin{array}{l}\text { - Trading post band } \\
\text { + Caza-recolección } \\
\text { + Dependencia } \\
\text { intercambio de bienes } \\
\text { europeos } \\
\text { + Jefe banda-intermediario }\end{array}$ & $\begin{array}{l}\text { + Reserva indígena } \\
\text { + Participación sistema } \\
\text { productivo industrial } \\
\text { + Dependencia economía } \\
\text { monetaria y de mercado } \\
\text { + Dependencia asistencial } \\
\text { + Consejo de banda } \\
\text { + } \\
\text { Autogobierno (1980) }\end{array}$ \\
\hline
\end{tabular}

Fuente: Marquina, Ruiz-Callado y Vírchez. Elaboración propia a partir de los datos etnohistóricos de Rogers y Smith (1994). 


\subsubsection{Agrupaciones familiares de caza (invierno) y bandas (verano)}

Antes de la definitiva sedentarización en las primeras décadas del siglo xx, los ancestros más cercarnos en el tiempo de Omushkego First Nation desarrollaron una organización social basada en pequeñas agrupaciones flexibles y subtribales. Estos grupos estaban formados por varias familias que cazaban de manera independiente durante gran parte del año. Mientras estos grupos familiares vivieron en el extenso bosque boreal subártico, fueron unidades de producción y consumo autónomo (Rogers, 1994; Bishop, 1994). Solo durante unos pocos meses al año, como consecuencia de las condiciones ecológicas y climatológicas, estos pequeños grupos familiares de cazadores-recolectores nómadas e independientes se reunían con otros grupos familiares subtribales para formar bandas de verano.

Durante los meses de octubre a abril se establecían campamentos de caza compuestos por varias familias nucleares (los hermanos y sus padres). Estas agrupaciones familiares tenían un íntimo conocimiento y vínculos históricos con un territorio de caza. Aunque este sistema social era el deseado por los grupos, no siempre era posible debido a muertes o divisiones familiares. En ese caso, los grupos de invierno podrían estar compuestos por cuñados o algún pariente más distante. Sin embargo, rara vez, un grupo de caza en invierno incluiría a un individuo sin vínculos de parentesco con algún miembro del grupo.

El liderazgo era adquirido de manera informal. El líder, normalmente un individuo que podía proporcionar comida u otras necesidades, adquiría la responsabilidad del grupo. Su estatus conllevaba tareas como aconsejar y asesorar en asuntos espirituales e intentaba proteger al grupo de cualquier problema causado por fuerzas naturales o sobrenaturales. Para lograr comunidades morales eficaces, una conducta jerárquica por parte de estos líderes hubiera dado como resultado fracturas y divisiones sociales. Estos grupos de caza necesitaban producir una ética igualitaria como mecanismo de supervivencia en un entorno tan hostil como el subártico canadiense. La hostilidad era suprimida en los grupos de caza para hacer frente al duro invierno y a la falta de alimentos. De esta manera, los cazadores más afortunados durante un invierno ofrecían parte de su carne a otros cazadores menos afortunados.

Durante los meses de verano, estos grupos de caza establecían centros tradicionales de reunión ceremonial. Los encuentros con otras familias que habían pasado el invierno esparcidas por el amplio territorio del norte de Ontario servían para celebrar rituales religiosos, reforzar lealtades políticas y sellar lazos de parentesco mediante matrimonios entre distintos grupos de caza. Como regla general estos grupos de caza, cada verano, o a veces en otros momentos del año, formaban una «banda» cuyos miembros estaban estrechamente unidos por vín- 
culos de parentesco. En el norte de Ontario, una banda constaba de entre 100 y 200 individuos. Estas bandas solían reconocer a un anciano como su líder. Sin embargo, como ocurría con los líderes de los grupos de caza, este cargo no tenía autoridad real, sino meramente una autoridad moral. Los líderes mantenían su estatus debido a su carisma y, por supuesto, a sus poderes sobrenaturales como chamanes y hombres-medicina. En cualquier caso, el líder ni era elegido ni heredaba el cargo. A su muerte, la banda podía dividirse y reagruparse en nuevas bandas. Durante el siglo xix este sistema social de liderazgo llegaría a ser alterado como resultado de la expansión de puestos de intercambio de pieles por todo el norte de Ontario. Para entonces una nueva organización social había surgido, la trading post band (Rogers y Smith, 1994).

\subsubsection{Trading post band: centros de intercambio comercial}

A finales del siglo xviII, la organización social de las poblaciones indígenas del norte de Ontario se había adaptado perfectamente a la economía de comercio de pieles. Por aquella época, esta región, y gran parte del oeste de Canadá (Alberta, Manitoba y Saskatchewan, además de Nunavut y los Territorios del Noroeste), pertenecía a la compañía comercial de la Bahía de Hudson. En el siglo xvir, esta compañía, fundada en 1670, había creado en las bahías de Hudson y de James tres puestos comerciales costeros: Moose Factory, Fort Albany y Fort Severn. Con el tiempo, estos tres centros comerciales contarían con trading-post bands, es decir, familias extendidas interrelacionadas entre sí que pasaban el invierno cerca de estos puestos, manteniendo relaciones comerciales para el intercambio de pieles por bienes europeos muy apreciados en aquel tiempo por las poblaciones indígenas (cuchillos, cazos, armas, pólvora...). El modo de vida seminómada fue paulatinamente reemplazado por un asentamiento central y permanente con mayor dependencia de los bienes manufacturados europeos, que ofrecía más oportunidades de alimento, atención sanitaria y empleo.

El origen contemporáneo de Omushkego Lake First Nation se remonta a un lugar denominado por las fuentes históricas Mammamattawa o meeting of waters ('encuentro de aguas'). Uno de los puestos más antiguos en el interior, el puesto Henley House de la compañía de la Bahía de Hudson, fue trasladado, a finales del siglo xVIII, a una nueva localización estratégica para comerciar con los grupos indígenas de la zona. Este nuevo puesto, situado en la confluencia de los ríos Kenogami, Nagagami y Kabinakagami, originó la creación de una banda vinculada al puesto comercial. Esta banda empezó a pasar más tiempo al lado del puesto de la compañía de la Bahía de Hudson durante sus reuniones de verano, socializando mediante actividades comerciales, rituales y alianzas políticas con grupos 
más grandes que las agrupaciones familiares nucleares extendidas sobre territorios tradicionales de caza. Los grupos indígenas cree y ojibwe en Mammamattawa llegaron a ser una banda home guard para el puesto, proporcionando servicios a la compañía de la Bahía de Hudson (Pollock, 2006).

Entre 1770 y 1880 , el deseo de adquirir bienes europeos entre las poblaciones indígenas cree y ojibwe, que habitaban el extenso territorio del norte de Ontario, llegó a ser mucho más intenso. Para estas poblaciones, el comercio de pieles resultó ser muy rentable, puesto que podían obtener fácilmente bienes manufacturados europeos altamente valorados por los indígenas con escaso esfuerzo. Esto era debido a la abundancia de animales, especialmente del castor, cuya piel era muy apreciada por los europeos, y a sus enormes habilidades como cazadores adquiridas durante siglos de vida en el bosque boreal. Cuando a finales del siglo xix las pieles de castor llegaron a ser escasas, los indígenas del norte tuvieron dificultades para adquirir más bienes europeos, dependiendo cada vez más de la «asistencia social» de los empleados y encargados de los puestos comerciales de la compañía de la Bahía de Hudson.

Aunque las poblaciones indígenas llegaron a ser la base de bandas flexibles e independientes, cada una asociada con un puesto comercial, la vida social de los cree y los ojibwe del norte de Ontario, probablemente, continuó siendo muy similar antes del contacto europeo hasta que, entre finales del siglo xix y principios del xx, el mercado de pieles se colapsó y su dependencia económica de bienes manufacturados europeos, fraguada durante los siglos anteriores, les empujó a entrar al emergente mundo industrial forestal, minero y de las grandes infraestructuras de comunicación terrestre, como vías ferroviarias y carreteras.

\subsubsection{Reserva indígena: sistema organizacional de tutela estatal}

A finales del siglo xix y principios del xx, la población en Ontario crecía y se concentraba en el sur. Esta presión demográfica generó un creciente interés por la colonización agrícola del remoto norte de Ontario y sus recursos naturales, y comenzó un periodo de negociaciones de tratados con comunidades indígenas para la cesión de sus tierras de caza a cambio de la creación de reservas y la promesa de viviendas, trabajo y asistencia social (Wood, 2000). El Tratado n. 9 o Tratado de Bahía James fue negociado con la banda instalada en Mammamattawa en 1905, y dio lugar a la creación administrativa de la English River Indian Reserve (Morrison, 1986).

Desde el punto de vista sociológico, el desplazamiento de campamentos de caza estacionales a la vida sedentaria y colectiva en hogares permanentes, con varios cientos de personas viviendo juntas, fue una experiencia relativamente nueva 
para los cree y los ojibwe del norte de Ontario. La experiencia social más cercana y equiparable había ocurrido durante el periodo poscontacto de comercio de pieles durante las reuniones comunales en los puestos de intercambio de las compañías comerciales durante los meses de verano. La estructura social tradicional de los grupos familiares de caza sería, gradualmente, debilitada en la vida sedentaria de la reserva, al tener poca relevancia práctica en la vida diaria del mundo industrial.

\subsection{Consecuencias sociales contemporáneas del sistema reserva}

En la década de 1950 una nueva conciencia pública emergió en Canadá respecto a las poblaciones indígenas, acelerando una política de sedentarización y de bienestar social (Warry, 1998; Dyck y Waldram, 1993). Esta política pública de asentamientos permanentes bajo la forma administrativa de reservas, que supuso la extensión del estado de bienestar a poblaciones indígenas justo cuando sus economías tradicionales de comercio de pieles estaban sufriendo un colapso, ha demostrado ser una de las políticas públicas más ineficaces en la historia canadiense (Flanagan, 2008; Carstens, 2000). En el debate sobre la estructura social contemporánea de las reservas se reitera que la estructura institucional de este tipo de formación socioadministrativa, diseñada por planificadores gubernamentales coloniales, ha alimentado el desempleo estructural, la dependencia de programas de asistencia social y el faccionalismo, produciendo desigualdad social en el interior de las reservas. Las reservas han llegado a representar, en el imaginario canadiense, los espacios sociales más devaluados y marginales. A continuación se examinarán las tres características estructurales que, identificadas en el estudio de caso objeto de este análisis, explicarían el aumento de problemas psicosociales y epidemias sociales como depresión, adicciones y violencia doméstica, que asolan las reservas indígenas del norte de Ontario y, por extensión, de Canadá.

\subsubsection{Desempleo estructural}

Los individuos contemporáneos de ascendencia indígena en Omushkego Lake First Nation ya no son excepcionales debido a sus actividades económicas, sus prácticas religiosas, su vida ceremonial o social, su estilo de asentamiento o la comida con la que se alimentan. La vida en la reserva es irreconocible respecto a aquella sociedad tradicional en la que todas las familias dependían de una economía de subsistencia cazadora-recolectora. La mayoría de los habitantes de Omushkego Lake First Nation viven sus vidas con un estilo muy similar al del resto de poblaciones rurales cercanas a la reserva: asisten al sistema educativo 
para ser alfabetizados y para adquirir las habilidades técnicas necesarias en la sociedad moderna canadiense; poseen propiedades individuales; trabajan a cambio de salarios remunerados; satisfacen sus necesidades mediante transacciones de mercado más que por autoprovisión de artículos del bosque (pieles, carne, utensilios...) y tratan con agencias estatales para cuestiones administrativas y legales. Por tanto, los individuos contemporáneos de ascendencia cree y ojibwe en Omushkego deben observarse como una variante subcultural de la clase trabajadora rural, con una clase media profesional emergente, en el marco de la estratificación social y estructura de clases de Canadá (Flanagan, 2008; Kuper, 2005). ${ }^{6}$

La creación de Omushkego Lake First Nation estuvo estrechamente ligada a la política provincial de la colonización agrícola del New Ontario y lo que se conoció en su momento como la Great Railroad Building Era (1881-1931). Tras el colapso del comercio de pieles, lugares como Mammamattawa sirvieron para reubicar la «fuerza de trabajo» cree y ojibwe que había trabajado en la industria ferroviaria durante las primeras décadas del siglo $\mathrm{xx}$, hacia la industria forestal emergente en el norte de Ontario. La colonización no era posible sin una infraestructura ferroviaria que abriera el norte a habitantes del sur de la provincia para repoblar una inmensa área virgen para la explotación agrícola y forestal. Tras el fallido intento político de atraer a colonos al norte de Ontario para crear una estructura económica agrícola, la década de 1940 convertiría parte del bosque boreal subártico en una región próspera debido al desarrollo de la industria forestal. Inmigrantes finlandeses, suecos, ucranianos, portugueses, irlandeses y francófonos procedentes de Quebec llegaron al Norte para trabajar en el bosque y en aserraderos.

El crecimiento y desarrollo de la industria forestal cerca de Mammamattawa tuvo lugar entre las décadas de 1940 y 1950 . En estas décadas se crea en la localidad ferroviaria de Calst una empresa familiar, Lecours Lumber Co. Limited,. Al mismo tiempo, el aislamiento geográfico de las comunidades en el norte de Ontario, a donde solo se podía acceder mediante tren, impulsó un plan de construcción de carreteras. En este contexto de expansión económica y de infraestructuras y ante la escasez de mano de obra en la región, el gobierno canadiense decidió que el lugar ideal para relocalizar la comunidad cree y ojibwe de Mammamattawa era junto al lago Constance, cerca de la localidad Calst.

La promesa de construcción de casas, junto a la perspectiva de trabajos asalariados, atrajo a muchos cree y ojibwe que residían en Mammamattawa a trasladarse a la nueva reserva en el lago Constance. La gran mayoría había finalizado

6 Actualmente, la reserva tiene un jefe y seis consejeros de banda que son elegidos colectivamente por un periodo de dos años, ejerciendo el tipo de autoridad formal que ejercían los agentes indios hasta la década de 1960. Aunque algunos ancianos hablan su idioma materno, la mayoría de la población en la reserva solo habla inglés. 
su trabajo en la infraestructura ferroviaria y el comercio de pieles estaba en crisis. La moderna reserva Omushkego Lake First Nation fue construida junto al lago Constance y la gran mayoría de los residentes empezó a trabajar para Lecours Lumber Co. y en la construcción de la carretera entre Grant y Calst. Sin embargo, la reserva fue establecida sobre la base de decisiones externas, por planificadores gubernamentales que no tuvieron en cuenta muchos aspectos de la vida diaria en el norte de Ontario.

El desempleo estructural ha sido una constante desde las décadas de 1950 y 1960, haciendo la vida en la reserva más difícil, puesto que los dos sectores económicos principales, la industria forestal y la construcción de carreteras, tenían una estructura laboral basada en el empleo estacional y esporádico, complicándose aún más en las décadas de 1970 y 1980 por la mecanización de la industria forestal, que reducía la necesidad de mano de obra. Como consecuencia de la escasez de empleo remunerado para los habitantes de la reserva y los costes monetarios para mantener los estándares de vida moderna, los residentes se hicieron cada vez más dependientes económicamente del estado canadiense. Además, la apertura de la reserva a carreteras dio a los miembros de la banda acceso a nuevos servicios, productos y bienes y la oportunidad de visitar otros lugares; ello conllevó una necesidad creciente de ingresos económicos a los que no todos podían tener acceso.

En 2006, la tasa de desempleo en las reservas indígenas era un 302\% más alta que en la provincia de Ontario y un 160\% mayor que en la región del norte de dicha provincia (Yahn, 2009). Por otro lado, las comunidades indígenas en el norte de Ontario tienen un porcentaje más alto de jóvenes, una población más baja de ancianos y niveles educativos más bajos que la media en las comunidades rurales del norte de Ontario (INAC, 2010). Por tanto, las oportunidades económicas en estas comunidades indígenas siguen siendo menores y se observa muchos más problemas estructurales que en el resto de las comunidades rurales del norte de Ontario, con tasas más altas de desempleo y un uso crónico de la asistencia social.

\subsubsection{Dependencia de programas de asistencia social}

Paulatinamente, la población indígena que reside en Omushkego First Nation estuvo, cada vez más, bajo la tutela de agencias gubernamentales, de modo que muchos adultos fueron incapaces de alcanzar las expectativas culturales de autonomía e independencia que el periodo industrial parecía prometer. La vida en la reserva significó la sumisión a una economía monetaria y de mercado, con la desventaja de residir en lugares remotos sin las alternativas laborales, recreacionales y de servicios de las que disponían las comunidades del sur más urbanizado 
de la provincia (Vírchez y Faucheux, 2012). Desde entonces, las reservas sufren tasas de desempleo muy elevadas y epidemias sociales como el alcohol y otras drogas. Desde el año 2005, el abuso de fármacos legales para fines recreativos, OxyContin y Percocet, ha llegado a ser una epidemia social en Omushkego, con cerca de 200 miembros asistiendo a un centro de metadona instalado en la reserva. Una encuesta, realizada en 2008 por el centro de salud en la reserva reveló que el 46,3\% de la población abusó de los fármacos legales mencionados arriba y un $36 \%$ declaró consumir regularmente drogas ilegales. Esta situación ha afectado a la estructura familiar y comunitaria, aumentando el nivel de violencia, robos, divorcios y otros problemas de convivencia (Chiefs of Ontario, 2010; Sioux Lookout, 2009).

Aunque el desarrollo de programas de bienestar social, financiados federalmente ha reducido la desigualdad de ingresos y de niveles de educación, este sistema público ha llegado a profundizar la autoimagen devaluada que seguían arrastrando las poblaciones indígenas durante décadas (Barsh, 2008). El sentimiento de exclusión, rechazo, desmoralización e impotencia está generalizado entre mucha gente de Omushkego Lake First Nation. Dixon y Scheurell (1995) indicaron que durante la década de 1980 y principios de 1990, la tasa de dependencia de la asistencia social para las poblaciones indígenas era cuatro veces mayor que la tasa nacional, y la tasa para indígenas que residían en reservas era seis veces mayor, atribuyendo estos resultados a la historia del mercado de pieles, la desposesión de la tierra y las políticas de asimilación e integración.

Desde la década de 1950 hasta la actualidad, el gasto público sobre la población de las reservas no ha dejado de crecer en Canadá. La dependencia asistencial ha ido creciendo década tras década. En 1966, cerca del 36\% de los habitantes de las reservas canadienses estaban recibiendo asistencia social. Desde esa fecha, la cifra ha seguido creciendo. En 1992, era del 42\% respecto al 10\% para otros ciudadanos canadienses. La tasa de dependencia del sistema de servicios sociales en la reserva era del $45 \%$ en 1998 y en la actualidad se estima que está por encima del 65\%. En reservas más remotas, de las bahías de James y Hudson, la tasa llega hasta el $80 \%$ de la población (Vírchez y Faucheux, 2012). Esta dependencia de los programas asistenciales ha estado desconectada de la economía productiva y la asistencia social ha continuado aumentando durante casi cuatro décadas tanto si la economía canadiense crecía como si estaba en recesión. Moscovitch y Webster (1995) concluyen que el bienestar producido por el sistema asistencial en las reservas ha llegado a ser un modo de vida, transmitido de generación a generación y a menudo racionalizado por los receptores como un derecho emanado de los tratados coloniales. 
En Omushkego Lake First Nation, como en cualquier reserva indígena, la figura jurídica especial de indian status produce un modo de vida, generado por un conjunto de estímulos sociales ambivalentes, que algunos investigadores han llamado la «subcultura de reserva» ${ }^{7}$ (Flanagan, 2008; Thatcher, 2004; Carstens, 2000). Como el resto de comunidades indígenas en el norte de Ontario, Omushkego Lake First Nation tiene un porcentaje más alto de jóvenes, desempleo y niveles educativos más bajos que el resto de comunidades rurales no indígenas del norte de Ontario. A menos que el joven tenga un nivel educativo suficiente o relaciones estrechas en la ciudad o comunidad rural cercana, es más rentable permanecer en la reserva sin empleo que acceder al mercado de trabajo ${ }^{8}$ Por ejemplo, los jóvenes que deciden abandonar la reserva y trasladarse a ciudades como Thunder Bay o comunidades rurales como Grant, entran a formar parte de los estratos más bajos de la estructura socioeconómica con un salario mínimo; tienen que pagar impuestos, rentar o comprar una vivienda y pierden las ayudas asistenciales (Ruiz-Callado, 2007). El estándar de vida en la ciudad o comunidad rural podría ser ligeramente superior al que disfrutan en Omushkego Lake First Nation, donde a pesar de la escasez de trabajo en las reservas, los jóvenes cuentan con una cobertura social básica de bienestar. La asistencia social ha llegado a ser una estrategia beneficiosa para los residentes más jóvenes de la reserva con escasa formación educativa, temerosos de experimentar la economía de mercado y la discriminación fuera de la reserva en contextos étnicos más plurales de la sociedad canadiense (Ruiz-Callado, 2005). Como resultado, muchos jóvenes de la reserva no desarrollan la mentalidad dominante en Canadá basada en la ética del esfuerzo e incentivo individual.

Actualmente, estas comunidades indígenas son vistas, desde el sur de la provincia de Ontario, como lugares residenciales decadentes, y se infravaloran por haber recibido cuantiosa financiación pública para compensar sus elevadas cifras de desempleo y sus numerosos problemas psicosociales.

\footnotetext{
7 La aprobación del Indian Act, actualmente en vigencia, supuso la creación de la figura jurídica del indian status. Indian status es el derecho que tiene una persona en Canadá cuyos ancestros firmaron alguno de los tratados que se llevaron a cabo durante los siglos XIX y Xx y cuyo nombre aparece en el Indian Register [registro indio] del Departamento Federal de Asuntos Indígenas. Tener indian status otorga a la persona el derecho a participar en las decisiones comunitarias, al usufructo de la tierra cedida a la reserva y a los servicios y programas gubernamentales. Jurídicamente, el indian status, residiendo en una reserva contemporánea, tiene derechos civiles y oportunidades especiales, y también desventajas, que cualquier canadiense no tiene: está exento de gravámenes fiscales sobre sus ingresos salariales o propiedades, la educación postsecundaria es completamente gratuita y los servicios sanitarios especializados son gratuitos, al igual que el acceso a la vivienda (Flanagan, 2008). Estos derechos especiales están sujetos a la residencia en la reserva como miembro afiliado.
}

8 Los jóvenes que adquieren educación formal, a menos que trabajen para el consejo de banda y sus ramas administrativas, tienden a marchar a la ciudad. 


\subsubsection{Faccionalismo y clientelismo político}

La vida en reservas como Omushkego Lake First Nation presenta una serie de particularidades dentro de la democracia liberal canadiense que la distancia socialmente del resto de comunidades rurales de las provincias de la federación canadiense. Cualquier canadiense no puede acceder libremente a Omushkego Lake First Nation, adquirir una propiedad o decidir establecerse en la reserva y residir allí permanentemente. Incluso, si alguien intenta, como investigador o periodista, aventurarse a explorar la reserva sin seguir cierto protocolo indígena ceremonial, puede ser físicamente expulsado por el consejo de banda. La memoria de dominación y opresión de la historia colonial entre el «hombre blanco» y el «indio» proporciona el telón de fondo para entender esta realidad social contemporánea.

Esta situación social, atípica en una democracia moderna como Canadá, es el resultado del contexto poscolonial de reclamación de tierras ancestrales y autogobierno que tuvo lugar en las décadas de 1970 y 1980. Omushkego Lake First Nation está implicada en un proceso de reconquista del derecho al autogobierno indígena para acabar con la desigualdad estructural de control institucional sobre la regulación administrativa de distintos ámbitos de la vida en la reserva. Hasta la década de 1960, los denominados agentes indios, sujetos a control administrativo por el Departamento de Asuntos Indígenas, ejercían una fusión extraordinaria de poderes judiciales, ejecutivos y legislativos sobre los residentes de las reservas. Aquellos poderes han sido ahora transferidos al jefe y al consejo de banda de Omushkego Lake First Nation.

Dentro de este contexto poscolonial de negociación política, revisión histórica y reconciliación nacional ha habido una transferencia progresiva de poder al gobierno local indígena de Omushkego Lake First Nation. Muchas reservas indígenas empezaron a negociar con el gobierno canadiense para exigir el reconocimiento del autogobierno indígena y la responsabilidad administrativa en el control de las transferencias de programas asistenciales y desarrollo económico. Más del 80\% de las transferencias de programas sociales gestionadas por el Departamento de Asuntos Indígenas del gobierno canadiense han sido cedidas a los consejos de banda para el autogobierno y autoadministración de servicios y programas federales y provinciales.

El autogobierno indígena en Omushkego Lake First Nation ha producido ciertos beneficios como, por ejemplo, un sentido de orgullo, autonomía y autoconfianza con la aspiración de un futuro de prosperidad. En Omushkego, se concibe el autogobierno en términos de asunción de la autoridad y la estructura institucional tradicionalmente mantenidas por el Departamento de Asuntos Indígenas. Desde un punto de vista simbólico de la acción política, en la reserva 
se ve el autogobierno como una manera de recuperar los patrones comunales de su pasado precolonial y deshacerse de las instituciones formales y burocráticas impuestas por el Acta India. Los líderes políticos de la comunidad defienden la transferencia federal de dinero público como reparación de errores coloniales anteriores y afirman que la inmunidad al sistema fiscal es una condición perpetua, un derecho indígena, un derecho de tratado colonial y un componente fundamental de la relación especial entre los indígenas y el estado canadiense.

Sin embargo, la transferencia de dinero público se ha traducido en la emergencia de una elite política y empresarial, bien situada en la reserva, y de organizaciones políticas y administrativas regionales. En un nivel inferior, una nueva clase profesional de administradores, abogados, consultores, maestros y trabajadores sociales ha encontrado empleo federal al administrar los programas gubernamentales que supuestamente deberían servir para facilitar el desarrollo económico. Esta situación estructural e institucional ha generado la polarización interna en la reserva, en la cual la conformación de minorías burocráticas, empresariales y profesionales se ha visto acompañada por el desempleo creciente y por la dependencia asistencial de la mayoría de habitantes. En este sentido, la reclamación de tierras ancestrales y autogobierno ha generado un sistema organizacional en la reserva que está alimentando una estructura institucional basada en un régimen político rentista y patrimonial (Vírchez y Faucheux, 2012). La transferencia fiscal de dinero público y poder ha aumentado los abusos de faccionalismo, nepotismo y clientelismo. La lucha de grupos de parentesco extendidos y redes de apoyo ha sido el motor de políticas internas en la reserva ${ }^{9}$ La autofinanciación de las reservas depende exclusivamente de transferencias gubernamentales, reclamaciones de tierra y royalties por la explotación de recursos en tierras ancestrales a empresas madereras o mineras. ${ }^{10}$

Por tanto, junto al desempleo estructural y la dependencia asistencial, otro problema inherente en la estructura organizacional de las reservas es la práctica del nepotismo y el clientelismo como principios estructurales clave de la estructura institucional. Los residentes ven la distribución de la financiación pública como el propósito principal de la política local. En grupos sociales como las reservas, los lazos personales y de amistad son extremadamente importantes y, desde

9 El faccionalismo no es exclusivo del mundo indígena. El faccionalismo en la política canadiense opera también a escala local en pequeñas comunidades y a escala nacional de un modo formalizado, implicando competición entre organizaciones económicas, regionales y lingüísticas. Por ejemplo, durante la realización de trabajo de campo en una comunidad cercana a la reserva, se observó un estilo de conducta política similar con grupos de parentesco, redes de amistad y afiliaciones religiosas compitiendo en la política local.

10 El consejo de banda es el principal empleador, administrando tanto instalaciones de servicios públicos como empresas públicas: Mamawmatawa Holistic Education Centre, Jane Mattinas Health Centre, Band Office, Nishnawbe Aski Police Service, Sunrise Elders Complex, Methadone Maintenance Program, Amik Logging, Weekoban trucking company. 
un punto de vista cultural, los miembros de las bandas reconocen abiertamente el parentesco como el principio fundamental de la política. En la reserva, a falta de partidos políticos formales, hay campañas informales que implican redes de amistad y de parentesco.

Varios estudios sociológicos señalan que las condiciones de vida se deterioran en la mayoría de las reservas para aquellos individuos que están fuera de la estructura de poder (Flanagan, 2008). El crecimiento de la población en las reservas intensificará la escasez de trabajos, de vivienda y de otros servicios fundamentales, de tal modo que cada vez más gente buscará nuevas oportunidades en las ciudades canadienses. A pesar del autogobierno, las reservas crecerán menos y serán menos relevantes para la mayoría de la población indígena de Canadá. Las poblaciones de ascendencia indígena llegarán a ser, de facto, un nuevo grupo étnico migrante en la sociedad pluralista canadiense. De hecho, los últimos estudios sobre dinámica poblacional señalan que algo más de la mitad de la población indígena reside fuera de las reservas (INAC, 2010).

\section{Conclusiones}

El propósito de este artículo ha sido explorar el sistema de reserva indígena canadiense contemporáneo bajo el concepto teórico de ingeniería social planificada, desarrollado en los últimos trabajos académicos de James Scott. Según el marco teórico de Scott, los esfuerzos estatales de planificación centralizada fracasan en su objetivo de mejorar la condición humana, al no considerar el conocimiento local práctico que acumulan los grupos sociales a los que se quiere aplicar las políticas de planificación técnica y administrativa. Scott aplica el concepto de alto modernismo a una variedad de esfuerzos estatales que ha conducido a lo largo del siglo $\mathrm{xx}$ a verdaderas tragedias humanas, a pesar de las buenas intenciones iniciales por lograr cierta prosperidad en las poblaciones. Según Scott, la confluencia desafortunada de tres elementos lleva al fracaso de la planificación centralizada: 1) el deseo de lograr un orden administrativo de la naturaleza y la sociedad, 2) la aspiración del estado moderno de utilizar el poder coercitivo para lograr el orden deseado y 3) la presencia de una sociedad civil débil que es incapaz de resistir el uso del poder por parte del estado. La creación del sistema de reserva indígena se puede contemplar como un ejemplo ilustrativo de ingeniería social contemporánea, es decir, como un mecanismo administrativo y de organización social con propósitos asimilacionistas. El objetivo de extender la ideología del 
alto modernismo a las poblaciones indígenas mediante la planificación estatal centralizada de asentamientos permanentes ha tenido consecuencias contradictorias y ambivalentes.

Paradójicamente, por un lado, el objetivo explícito del modernismo liberal de introducir el «bienestar social» en las poblaciones indígenas a través de trabajo remunerado, la educación pública, la sanidad y los servicios sociales ha generado una infraclase socioeconómica en un país como Canadá, que mantiene uno de los índices de desarrollo humano más elevados del mundo. Por otro lado, las políticas poscoloniales de reconciliación nacional y la conquista de mayor autogobierno indígena están produciendo como resultado una emergente división de clase social: una clase social profesional indígena bien remunerada y con aspiraciones sociopolíticas, y una clase social trabajadora, sin estudios, con trabajos estacionales en la industria forestal y minera y fuertemente dependiente de programas de asistencia social. Por lo tanto, el sistema de reserva indígena como estructura organizativa tiene problemas inherentes y contradictorios cuyos efectos están llegando a ser más visibles en reservas indígenas como Omushkego Lake First Nation. El sistema de reserva indígena está asolado con características estructurales que animan más que constriñen la desigualdad en las reservas. El mantenimiento de la estructura institucional de esta organización social está alimentando el desempleo estructural, la dependencia de programas de asistencia social y el faccionalismo, produciendo desigualdad social en el interior de las reservas.

\section{Bibliografía}

Adelson, N. (2004). «Being Alive Well»: Health and the Politics of Cree WellBeing. Toronto: University of Toronto Press.

Barsh, R. (2008). Canada's Aboriginal Peoples: Social Integration or Desintegration? Alberta: University of Lethbridge.

Berman, T. (2003). Circle of Goods: Women, Work, and Welfare in a Reservation Community. Albany: State University of New York Press.

Bishop, C. (1994). «Northern Algonquians», en Edward Rogers y Donald Smith (coords.). Aboriginal Ontario. Historical Perspectives on the First Nations. Toronto: Dundurn Press.

Bones, R. (2003). The Geography of the Canadian North. Oxford: Oxford University Press.

Brady, M. (1995). «Culture in treatment, culture as treatment. A critical appraisal of developments in addictions programs for indigenous North Americans and Australians». Social Science and Medicine, 41: 1487-1498. 
Carstens, P. (2000). «An Essay on Suicide and Disease in Canadian Indian Reserves: Bringing Durkheim Back in». The Canadian Journal Studies 20 (2): 309-345.

Chiefs of Ontario (2010). Take a stand. Prescription Drug Abuse Strategy Final Report. Toronto: Chiefs of Ontario.

Coates, K. (2008). The Indian Act and the Future of Aboriginal Governace in Canada. National Centre for First Nation Governance.

Ken y Morrison, W. (1992). The forgotten North: A bistory of Canada's provincial Norths. Toronto: J. Lorimer.

Dyck, Noel y Waldram, J. B. (Coord.) (1993). Anthropology, Public Policy, and Native Peoples in Canada. Montreal\&Kingston: University Mc-Gill-Queen Press.

Dixon, John y Scheurell, R. (1995). Social Welfare with Indigenous Peoples. London: Routledge.

Dow, F. (2008). «Envisioning Sustainable Forestry Communities in Northern Ontario». Tesis Máster, University of Waterloo.

Fast, P. (2002). Northern Athabascan survival: Women, community, and the future. Lincoln, NE: University of Nebraska Press.

Flanagan, T. (2008). First Nations? Second Thoughts. Montrealः McGill-Queen's University Press.

Health Canada (2002). A Report on Mental Illnesses in Canada. Ottawa: Public Health Agency of Canada.

Indian and Northern Affairs Canada [INAC] (2010). Registered Indian

Population by Sex and Residence. Ottawa: Department Aboriginal Affairs and Northern Development Canada.

Jones, D. (2004). Rationalizing Epidemics: Meanings and Uses of American Indian Mortality since 1600. Cambridge: Harvard University Press.

King, M.; Smith, A., y Gracey, M. (2009). «Indigenous health part 2: the underlying causes of the health gap». The Lancet, 374: 76-85.

Kirmayer, L. y Valaskakis, G. (Coord.) (2008). Healing Traditions: The Mental Health of Aboriginal Peoples in Canada. Vancouver: University of British Columbia Press.

Kuper, A. (2005). The Reinvention of Primitive Society: Transformations of a Myth. London: Routledge.

LaPierre, L. (1992). Canada, my Canada. What Happened? Toronto: McClelland y Stewart.

Marquina, A.; Ruiz-Callado, R., y Vírchez, J. (en prensa) (2012). «Envionmental Health, Indigenous Identity, Cultural Representations 
and the Production of Ecological Conscioussness in Northern Ontario». International Journal of Sustainable Development.

- (2012). La cultura herida: «Now I Know Who I Am, I Am Anishinaabe». Un estudio fenomenológico y sociolingüistico sobre salud mental, cognición cultural $y$ adicciones en una reserva indígena en el noreste de Ontario, Canadá. Tesis Doctoral. Universidad de Granada.

Mawhiney, A. M. y Pitblado, J. (1999). Boom Town Blues. Elliot Lake: Collapse and Revival in a Single-Industry Community. Toronto, Dundurn Press.

Morrison, J. (1986). Treaty No 9 (1905-1906). Treaties and Historical Research. Centre and Northern affairs Canada.

Moscovitch, A. y Webster, A. (1995). «Aboriginal Social Assistance Expenditures», en Susan D. Phillips (Coord.), How Ottawa Spends 19951996: Mid-Life Crises. Ottawa: Carleton University Press.

Parfitt, B. (2006). Public Forests, Public Returns. Vacouver: Canadian Centre for Policy Alternatives.

Pollock, J. (2006). Archaeological and Cultural Heritage Impact Assessment of the Sandy Falls. Toronto: Woodland Heritage Services.

Rogers, E. (1994). «Northern Algonquians and the Hudson's Bay Company, 1821-1890», en Edward Rogers y Donald Smith (Coord.) Aboriginal Ontario. Historical Perspectives on the First Nations. Toronto: Dundurn Press.

- y Smith, Donald (coord.) (1994). Aboriginal Ontario. Historical Perspectives on the First Nations. Toronto: Dundurn Press.

Ruiz-Callado, Raúl (2007). «Procesos de integración y precariedad de los jóvenes inmigrantes en las sociedades de acogida». Sociedad y Utopía, 30 : 51-64.

- (2005). «Modos de inclusión social de los jóvenes inmigrantes: la integración como fundamento de la ciudadanía democrática». Sociedad y Utopía, 26: 279294.

Scoтt, James (1998). Seeing Like a State: How Certain Schemes to Improve the Human Condition Have Failed. New Haven, CT: Yale University Press.

- (2009). The Art of Not Being Governed: An Anarchist History of Upland Southeast Asia. New Haven, CT: Yale University Press.

Sherrill, Grace (2002). Canada and the Idea of North. Montreal: McGillQueen's University Press.

Sioux Lookout First Nations Health Authority (2009). Chiefs forum on social issue: answering the call for belp: reducing prescription drug abuse in our communities. Sioux Lookout, ON: Sioux Lookout First Nations Health Authority. 
Tait, C. y Simpson, C. (2008). «The Mental Health of Aboriginal Peoples in Canada: Transformations of Identity and Community», en Kirmayer, L. Y Valaskakis, G. (coord.) Healing Traditions: The Mental Health of Aboriginal Peoples in Canada. Vancouver: University of British Columbia Press.

TAnner, A. (2008). «The origins of Northern Aboriginal social pathologies and the Quebec Cree Healing Movement», en Laurence Kirmayer y Gail Valaskakis, (coord.) Healing Traditions: The Mental Health of Aboriginal Peoples in Canada. Vancouver: University of British Columbia Press.

TAYlOR, G. (1994). «Northern Algonquians on the Frontiers of "New Ontario", 1890-1945», en Edward Rogers y Donald Smith (coord.) Aboriginal Ontario. Historical Perspectives on the First Nations. Toronto: Dundurn Press. Thatcher, R. (2004). Fighting Firewater Fictions: Moving beyond the Disease Model of Addiction in First Nations. Toronto: Toronto University Press.

Vírchez, J. y Faucheux, M. (2011). «Life in the James Bay Lowland. Past, Present and Future». Revista Mexicana de Estudios Canadienses, 22: 29-53.

- y Brisbois, Ronald (2007). "A Historical and Situational Summary of Relations Between Canada and the First Nations: The Case of the Community of Kashechewan in Northern Ontario». Revista Mexicana de Estudios Canadienses, 14: 87-100.

Wadden, M. (2008). Where the Pavement Ends. Vancouver: Douglas y McIntyre. Waldram, J*; Herring, A. y Young, K. (2006). Aboriginal health in Canada: Historical, cultural and epidemiological perspectives. Toronto: University of Toronto Press.

- (2004). Revenge of the Windigo: The construction of the mind and mental health of North American Aboriginal peoples. Toronto: University of Toronto Press. Warry, W. (1998). Unfinished Dreams: Community Healing and the Reality of Aboriginal Self-Government. Toronto: University of Toronto Press.

Wood, D. (2000). Making Ontario: Agricultural Colonization and Landscape ReCreation Before the Railway. Montreal: McGill-Queen's University Press. Woodman, D. (2010). Intersection and Identity in Two Forest Dependent Communities in Northern Ontario. Tesis Doctoral. University of Guelph. YAHN, J. (2009). Impact of the Ontario Works Program on the Quality of Life of Aboriginal People Living on Reserve in Northern. Tesis Doctoral. Lakehead University. 